\title{
A classification of duct modes based on surface waves
}

\author{
Sjoerd W. Rienstra* \\ Department of Mathematics and Computing Science, Eindhoven University of Technology, Eindhoven, The Netherlands \\ Received 15 February 2002; accepted 10 May 2002
}

\begin{abstract}
For the relatively high frequencies relevant in a turbofan engine duct, the modes of a lined section may be classified in two categories: genuine acoustic 3D duct modes resulting from the finiteness of the duct geometry, and 2D surface waves that exist only near the wall surface in a way essentially independent of the rest of the duct. Per frequency and circumferential order there are at most four surface waves. They occur in two kinds: two acoustic surface waves that exist with and without mean flow, and two hydrodynamic surface waves that exist only with mean flow. The number and location of the surface waves depends on the wall impedance $Z$ and mean flow Mach number. When $Z$ is varied, an acoustic mode may change via small transition zones into a surface waves and vice versa.

Compared to the acoustic modes, the surface waves behave-for example as a function of the wall impedance-rather differently as they have their own dynamics. They are therefore more difficult to find. A method is described to trace all modes by continuation in $Z$ from the hard-wall values, by starting in an area of the complex $Z$-plane without surface waves.
\end{abstract}

(C) 2002 Elsevier Science B.V. All rights reserved.

\section{Introduction}

Although a straight duct with uniform velocity profile and impedance wall is a simplification, it is an important model of the lined duct of a real turbofan engine [1-8].

The relatively easy analytic description of the sound field by means of modes provides much more insight in global trends like the effects of mean flow, frequency and impedance on the modal decay rates than any other, more "exact" numerical solutions. This remains true for slowly varying ducts where the modal concept is still applicable $[9,10]$.

A further understanding of the modal behaviour is therefore important for both interpretation and understanding of more complex sound fields. Particularly welcome is a better predicted behaviour of a mode's physically most distinctive property, the axial wave number. This is the aim of the present paper.

The essence of the behaviour to be described below is the same for any lined flow duct of constant cross-section. For simplicity, we will restrict our analysis to the prototype case of a hollow cylindrical duct.

\footnotetext{
* Tel.: +31-40-247-4603; fax: +31-40-244-2150.
}

E-mail address: s.w.rienstra@tue.nl (S.W. Rienstra). 


\section{Physical model}

We consider a circular symmetrical duct of radius $a$ with a compressible inviscid perfect gas flow, in dimensional form described by density $\tilde{\rho}$, pressure $\tilde{p}$, velocity $\tilde{\mathbf{v}}$, entropy $\tilde{s}$, and soundspeed $\tilde{c}$, satisfying

$$
\frac{\mathrm{d}}{\mathrm{d} \tilde{t}} \tilde{\rho}=-\tilde{\rho} \tilde{\nabla} \cdot \tilde{\mathbf{v}}, \quad \tilde{\rho} \frac{\mathrm{d}}{\mathrm{d} \tilde{t}} \tilde{\mathbf{v}}=-\tilde{\nabla} \tilde{p}, \quad \frac{\mathrm{d}}{\mathrm{d} \tilde{t}} \tilde{s}=0,
$$

where

$$
\tilde{s}=C_{V} \log \tilde{p}-C_{P} \log \tilde{\rho}, \quad \tilde{c}^{2}=\gamma \frac{\tilde{p}}{\tilde{\rho}} .
$$

$C_{V}$ is the heat capacity or specific heat at constant volume and $C_{P}$ is the heat capacity or specific heat at constant pressure. $\mathcal{R}$ is the specific gas constant and $\gamma$ the Poisson ratio, which are practically constant and given by $\mathcal{R}=C_{P}-C_{V}=286.73 \mathrm{~J} / \mathrm{kg} \mathrm{K}$, and $\gamma=C_{P} / C_{V}=1.4$ (for air).

This flow consists of a uniform mean flow with small perturbations given by

$$
\tilde{\mathbf{v}}=U_{0} \mathbf{e}_{x}+\mathbf{v}^{\prime}, \quad \tilde{p}=p_{0}+p^{\prime}, \quad \tilde{\rho}=\rho_{0}+\rho^{\prime} .
$$

The entropy perturbation $s^{\prime}$ is proportional to $p^{\prime}-c_{0}^{2} \rho^{\prime}$. The mean flow quantities $p_{0}, \rho_{0}, s_{0}, c_{0}$ are positive, the velocity $U_{0}$ is zero or positive, but less than $c_{0}$. Upon linearisation this yields

$$
\begin{aligned}
& \left(\frac{\partial}{\partial \tilde{t}}+U_{0} \frac{\partial}{\partial \tilde{x}}\right) \rho^{\prime}+\rho_{0} \nabla \cdot \mathbf{v}^{\prime}=0, \\
& \rho_{0}\left(\frac{\partial}{\partial \tilde{t}}+U_{0} \frac{\partial}{\partial \tilde{x}}\right) \mathbf{v}^{\prime}+\nabla p^{\prime}=0 \\
& \left(\frac{\partial}{\partial \tilde{t}}+U_{0} \frac{\partial}{\partial \tilde{x}}\right)\left(p^{\prime}-c_{0}^{2} \rho^{\prime}\right)=0 .
\end{aligned}
$$

By taking the curl of Eq. (1b), we find for the vorticity perturbations $\boldsymbol{\xi}^{\prime}=\nabla \times \mathbf{v}^{\prime}$

$$
\left(\frac{\partial}{\partial \tilde{t}}+U_{0} \frac{\partial}{\partial \tilde{x}}\right) \xi^{\prime}=0
$$

By combining Eqs. (1a)-(1c), we can derive a convected wave equation for the pressure

$$
\left(\frac{\partial}{\partial \tilde{t}}+U_{0} \frac{\partial}{\partial \tilde{x}}\right)^{2} p^{\prime}-c_{0}^{2} \nabla^{2} p^{\prime}=0 .
$$

From Eqs. (1c) and (1d), we see that in uniform flow entropy and vorticity perturbations are either identically zero, or just convected by the mean flow (i.e. $\partial / \partial \tilde{t}+U_{0} \partial / \partial \tilde{x} \equiv 0$ ). Note that in uniform flow pressure, vorticity and entropy are decoupled, so we can leave here any vorticity or entropy perturbations unspecified and consider only the pressure field.

We assume time harmonic perturbations with frequency $\tilde{\omega}$, and make dimensionless as follows:

$$
\mathbf{x}=\frac{\tilde{\mathbf{x}}}{a}, \quad t=\frac{c_{0} \tilde{t}}{a}, \quad \omega=\frac{\tilde{\omega} a}{c_{0}}, \quad p^{\prime}=\rho_{0} c_{0}^{2} \operatorname{Re}\left(p \mathrm{e}^{\mathrm{i} \omega t}\right), \quad \mathbf{v}^{\prime}=c_{0} \operatorname{Re}\left(\mathbf{v} \mathrm{e}^{\mathrm{i} \omega t}\right) .
$$

We introduce the mean flow Mach number $M=U_{0} / c_{0}(0 \leq M<1)$, and Eqs. (1e) and (1b) become the convected reduced wave (or Helmholtz) equation in $p$ and a relation with $\mathbf{v}$

$$
\left(\mathrm{i} \omega+M \frac{\partial}{\partial x}\right)^{2} p-\nabla^{2} p=0
$$




$$
\left(\mathrm{i} \omega+M \frac{\partial}{\partial x}\right) \mathbf{v}+\nabla p=0
$$

where

$$
\nabla^{2}=\frac{\partial^{2}}{\partial x^{2}}+\frac{\partial^{2}}{\partial r^{2}}+\frac{1}{r} \frac{\partial}{\partial r}+\frac{1}{r^{2}} \frac{\partial^{2}}{\partial \theta^{2}} .
$$

To the mean flow, the duct is hard walled, but for the acoustic field the duct is lined with an impedance wall, which means that the ratio of the complex amplitudes of the time harmonic pressure and normal velocity (directed into the wall) at the wall is prescribed: $p=Z v$, where $v$ is the radial component of $\mathbf{v}$ and the dimensionless complex number $Z$ is the specific impedance of the wall.

However, with flow we have to be careful. In the limit of vanishing viscosity, the boundary layer reduces to a vortex sheet, but the velocity perturbations $a t$ the wall remain different from the ones near the wall, being at different sides of the vortex sheet. (The pressure is continuous.) So when we apply the impedance wall boundary condition to the acoustic field in the flow, we have to include the kinematic effect of the vortex sheet. This modification was for the first time correctly given for uniform mean flow along a plane wall by [11], and later generalised for flow along curved surfaces by [12].

In the present notation, the impedance wall boundary condition with uniform mean flow is found as follows. If the position of the perturbed vortex sheet is given by

$$
r=1+\operatorname{Re}\left(\eta(x, \theta) \mathrm{e}^{\mathrm{i} \omega t}\right),
$$

continuity of streamlines yields the radial velocity $v$ on the flow side and the radial velocity $v_{w}$ on the liner side being given by (after linearisation)

$$
v=\left(\mathrm{i} \omega+M \frac{\partial}{\partial x}\right) \eta, \quad v_{w}=\mathrm{i} \omega \eta
$$

Since the pressure across the vortex sheet is continuous and by definition the impedance boundary condition at the wall is

$$
p=Z v_{w},
$$

we thus have after elimination of $\eta$

$$
\mathrm{i} \omega v=\left(\mathrm{i} \omega+M \frac{\partial}{\partial x}\right)\left(\frac{p}{Z}\right), \quad \text { while } \quad p=0 \text { if } Z=0, \quad \text { at } r=1 .
$$

Note that this equation remains valid for $M=0$.

\section{Duct modes}

Because of the circular symmetry, the general solution of Eq. (2a) is given by the Fourier-Bessel modal sum [1]

$$
p=\sum_{m=-\infty}^{\infty} \sum_{\mu=-\infty}^{\infty} A_{m \mu} J_{m}\left(\alpha_{m \mu} r\right) \mathrm{e}-\mathrm{i} m \theta-\mathrm{i} k_{m \mu} x,
$$

where $J_{m}$ is the $m$ th order Bessel function of the first kind [13], $m=0, \pm 1, \pm 2, \ldots, \mu= \pm 1, \pm 2, \ldots$, and $A_{m \mu}$ are modal amplitudes. Since $J_{-m}=(-1)^{m} J_{m}$, it is sufficient to consider here positive $m$ only.

Any radial modal wave number $\alpha$ is related to the corresponding axial modal wave number $k$ by the dispersion relation ( $\alpha$ and $k$ are dimensionless)

$$
\alpha^{2}+k^{2}=(\omega-M k)^{2} .
$$


The boundary condition (3) is satisfied by applying the condition to each mode, and assuming uniform convergence ${ }^{1}$ of series (4) near $r=1$. This leads to the eigenvalue equation

$$
(\omega-M k)^{2} J_{m}(\alpha)=\mathrm{i} \omega Z \alpha J_{m}^{\prime}(\alpha)
$$

which has a countable number of solutions $k=k_{m \mu}$ in the complex $k$-plane. The solutions do not depend on the chosen branch of $\alpha=\alpha(k)$ because the combination $\alpha J_{m}^{\prime}(\alpha) / J_{m}(\alpha)$ is a meromorphic function of $\alpha^{2}$. Note that Eq. (6) is also valid for the problem with zero mean flow. The governing equations remain linear.

\subsection{Propagation direction}

The modes are counted such that for $\mu>0$ they propagate in positive $x$-direction, and for $\mu<0$ they propagate in negative direction.

As the impedance wall absorbs acoustic energy if $\operatorname{Re}(Z)>0$, the modes will usually decay when propagating away from their source. Therefore, the propagation direction of most modes is found by considering the sign of $\operatorname{Im}\left(k_{m \mu}\right)$. If this is negative, the mode decays in positive direction and the mode is right-running. If it is positive the mode is left-running.

If the wall is not dissipative, for example if $Z=\infty$ (hard wall) or $Z$ is purely imaginary, some modes may have a real axial wave number, which means that $\operatorname{Im}\left(k_{m \mu}\right)=0$ and there is no direction of decay. Probably the easiest approach in this case is to take a suitable limit in $Z$, starting from a dissipative situation. Alternatively, without mean flow it is possible to consider the sign of the modal phase velocity ${ }^{2} \omega / k_{m \mu}$, but with flow the effect of convection (leading to the reduced axial wave number; see below) should be accounted for.

Although the sign of $\operatorname{Im}\left(k_{m \mu}\right)$ is the most common parameter to determine the propagation direction, there are some subtle problems in the case with mean flow, where the absorption of acoustic energy by the liner may be compensated by acoustic energy supplied by the mean flow vortex sheet. These problems are only partly solved $[14,15]$, and we will mention them here.

A vortex sheet separating two regions of mean flow with different velocity is unstable, the well-known KelvinHelmholtz instability. A vortex sheet along a solid wall, on the other hand, is not unstable, because the wall inhibits any motion normal to the wall. However, if the wall is not solid, like an impedance wall, and at the same time the wall is not absorbing too much energy, the vortex sheet seems to be unstable again for certain combinations of impedance and Mach number. This instability appears mathematically like a mode as in Eq. (4), but now increasing rather than decaying. At first sight, an increasing instability cannot be distinguished from a decaying regular acoustic mode propagating in opposite direction. Therefore, one of the found modes may have to be interpreted as an instability.

In [15] (see Eqs. (13a)-(13d)), it was shown that under certain conditions this instability can be recognised explicitly. If the frequency $\omega$ is high enough such that the geometry becomes essentially $2 \mathrm{D}$, the Mach number $M$ is low enough, and the impedance $Z$ as a function of $\omega$ is sufficiently simple (for example, a mass-spring-damper system $Z(\omega)=r+\mathrm{i} a \omega-\mathrm{i} b / \omega)$, the instability can be recognised analytically, by arguments of causality and continuation in the complex $\omega$-plane. We have no doubt that it is possible to generalise this for more arbitrary cases, but this has to be done numerically.

\section{Analysis}

The problem we will be dealing with is a thorough analysis of the behaviour of the axial wave numbers $k_{m \mu}$, defined by Eqs. (5) and (6), as a function of $Z$ and $M$ in the context of relatively high frequencies occurring in

\footnotetext{
${ }^{1}$ For $x \neq 0$, this is not a very stringent condition, as the convergence is greatly accelerated by the exponential, $\operatorname{since} \operatorname{Im} k_{m \mu}=\mathcal{O}(\mu), \mu \rightarrow \infty$.

${ }^{2}$ Considering the modal group velocity $\left(\mathrm{d} k_{m \mu} / \mathrm{d} \omega\right)^{-1}$ has not yet been shown to be productive. For example, an inevitable problem is that $Z$ is always $\omega$-dependent.
} 


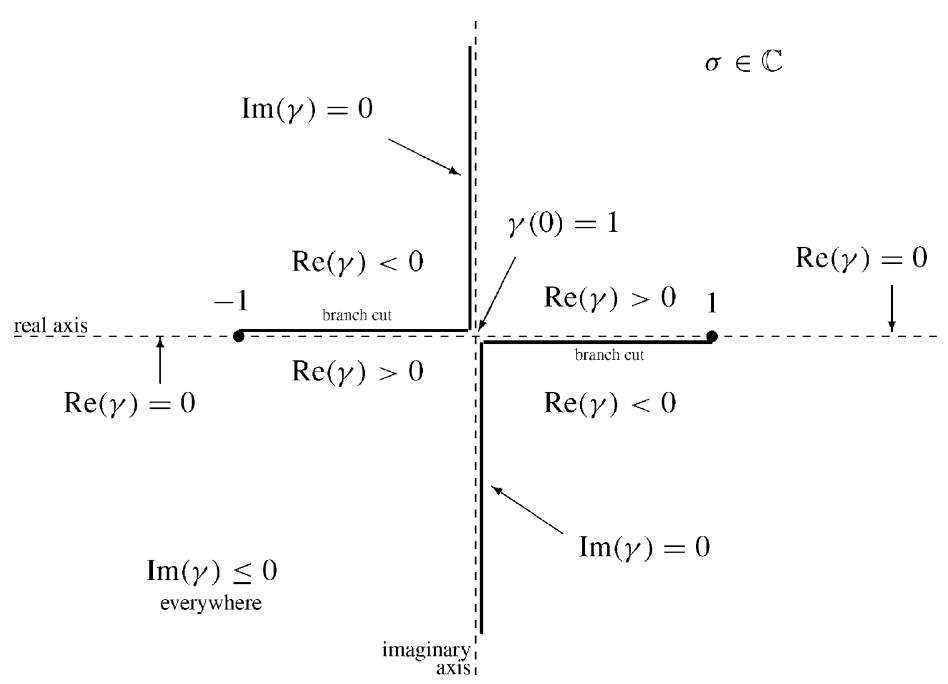

Fig. 1. Branch cuts and signs of $\gamma=\sqrt{1-\sigma^{2}}$ in complex $\sigma$-plane. The definition of $\gamma(\sigma)$ adopted here is the branch of the multi-valued complex square root that corresponds to $\operatorname{Im}(\gamma) \leq 0$ for all $\sigma \cdot \operatorname{Im}(\gamma)=0$ along the branch cuts. $\gamma(\sigma) \simeq-\mathrm{i} \sigma \operatorname{sign}(\operatorname{Re} \sigma)$ if $|\sigma| \gg 1$.

aircraft engine ducts. For this, it is convenient to introduce the Lorentz or Prandtl-Glauert type transformation that will render the equations as clean as possible. With $^{3}$

$$
\beta=\sqrt{1-M^{2}}, \quad x=\beta X, \quad \omega=\beta \Omega, \quad \alpha=\Omega \gamma, \quad k=\frac{\Omega}{\beta}(\sigma-M),
$$

(where in the rest of the paper $X=\operatorname{IM}(Z)$ ), the mode

$$
\mathrm{e}^{\mathrm{i} \Omega M X} \mathrm{e}^{-\mathrm{i} \Omega \sigma X} J_{m}(\Omega \gamma r)
$$

is scaled such that the asymmetry due to convection is now brought outside the expression as just a factor. The variables $\sigma$ and $\gamma$ are called the reduced axial and radial wave numbers. The dispersion relation and the eigenvalue equation is now

$$
\begin{aligned}
& \gamma^{2}+\sigma^{2}=1, \\
& (1-M \sigma)^{2} J_{m}(\Omega \gamma)=\mathrm{i} \beta^{3} Z \gamma J_{m}^{\prime}(\Omega \gamma),
\end{aligned}
$$

yielding infinitely many complex values of $\gamma$ and $\sigma$, depending on the parameters $M, Z, \omega$ and $m$.

Important special cases are: (a) the hard wall $Z=\infty$ with solutions $\Omega \gamma= \pm j_{m \mu}^{\prime}$ (the zero's of $J_{m}^{\prime}$ ), and (b) the pressure release wall $Z=0$ with solutions $\Omega \gamma= \pm j_{m \mu}$ (the zero's of $J_{m}$ ), or the additional solution $\sigma=M^{-1}$. Note that this last solution refers to the velocity field only, and not to the pressure, because for $Z=0$ this is zero at the wall and therefore zero everywhere.

To define the complex function $\gamma(\sigma)=\sqrt{1-\sigma^{2}}$ uniquely, we have to introduce branch cuts and select a branch. As in any wave problem, the sign of the imaginary part of a wave number is of primary importance (it selects decaying from increasing waves), we choose the branch cuts along the lines where $\operatorname{Im}(\gamma)=0$. Then we select the branch with $\operatorname{Im}(\gamma) \leq 0$, by defining $\gamma(0)=1$. See Fig. 1 .

\footnotetext{
${ }^{3}$ If $\kappa_{x}, \kappa_{r}$, and $\kappa_{0}=\tilde{\omega} / c_{0}$ indicate (respectively) the dimensional axial, radial, and free field wave numbers, then $\gamma=\beta \kappa_{r} / \kappa_{0}$ and $\sigma=$ $M+\beta^{2} \kappa_{x} / \kappa_{0}$.
} 


\subsection{No mean flow}

Without mean flow, i.e. $M=0$, Eqs. (8a) and (8b) simplify to

$$
\begin{aligned}
& \gamma^{2}+\sigma^{2}=1, \\
& J_{m}(\omega \gamma)=i Z \gamma J_{m}^{\prime}(\omega \gamma) .
\end{aligned}
$$

Now we can distinguish two important classes of solutions: a class with $\operatorname{Im}(\omega \gamma)$ large (in a sense to be explained below), and the complementary class. If $\operatorname{Im}(\omega \gamma)$ is large negative, the Bessel functions simplify to increasing exponentials (Eq. (A.1)), such that $J_{m}^{\prime} / J_{m} \rightarrow \mathrm{i}$, and the eigenvalue Eq. (9b) reduces to

$$
1+Z \gamma=0
$$

with solutions

$$
\sigma= \pm \sqrt{1-Z^{-2}} .
$$

As $\gamma$ was defined with negative imaginary part, the asymptotics of $\operatorname{Im}(\omega \gamma)$ large positive does not occur. At the same time, from this restriction on $\gamma$ it follows immediately that we have only such solutions if $\operatorname{Im}(Z) \leq 0$.

If $\omega$ is large, which is the typical situation in aircraft engine duct problems, any solution (10b)—except for nearly real impedances-produces a large $\operatorname{Im}(\omega \gamma)$ and is therefore an approximation of a solution of (9a) and (9b).

The physical interpretation of these solutions is that the corresponding mode is spatially confined to the immediate neighbourhood of the wall, as the modal shape function $J_{m}(\omega \gamma r)$ becomes exponentially decaying away from the wall (Eq. (A.1)):

$$
\left|\frac{J_{m}(\omega \gamma r)}{J_{m}(\omega \gamma)}\right| \simeq \frac{\mathrm{e}^{\omega \operatorname{Im}(\gamma)(1-r)}}{r^{1 / 2}} .
$$

In other words, these modes are not really duct modes, but surface waves. From Eq. (10b), it follows that there are at most two such surface waves, and they exist only if $\operatorname{Im}(Z)<0$.

This implies that the other possible solutions of Eqs. (9a) and (9b), the ones with $\operatorname{Im}(\omega \gamma)$ not large, necessarily occur near (i.e. typically within a distance $\mathcal{O}\left(\omega^{-1}\right)$ ) the branch cuts of square $\operatorname{root} \gamma(\sigma)$, where $\operatorname{Im}(\gamma)=0$.

Trajectories of these surface wave numbers, given by Eq. (10b), as function of $Z$, are plotted in Fig. 2. To include all complex values of $Z$, we have drawn two fan-shaped families of curves: one for fixed $\operatorname{Re}(Z)$ and one for fixed $\operatorname{Im}(Z)$, all of course with $\operatorname{Im}(Z) \leq 0$. Note that un-attenuated waves occur only for purely imaginary $Z$.

Trajectories of all duct mode eigenvalues are plotted in Fig. 3, as a function of the imaginary part $X$ of impedance $Z=R+\mathrm{i} X$, and various $R$, for $\omega=5$, and $m=1$. For illustration, the 2D surface wave approximations are included, and the agreement is seen to be very good. In fact, if we used a much higher $\omega$, the agreement would have been too good, with hardly any visible difference. This figure is further discussed in Section 5 . The reason why we let $\operatorname{Im}(Z)=X$ increase is explained in Section 6 .

\subsection{With mean flow}

With mean flow, i.e. $M>0$, we have to deal with the full equations

$$
\begin{aligned}
& \gamma^{2}+\sigma^{2}=1, \\
& (1-M \sigma)^{2} J_{m}(\Omega \gamma)=\mathrm{i} \beta^{3} Z \gamma J_{m}^{\prime}(\Omega \gamma) .
\end{aligned}
$$

Again, we can distinguish two important classes of solutions: a class with $\operatorname{Im}(\Omega \gamma)$ large, and the complementary class. If $\operatorname{Im}(\Omega \gamma)$ is large negative, $J_{m}^{\prime} / J_{m} \rightarrow \mathrm{i}$ (Eq. (A.1)), and the eigenvalue Eq. (8b) reduces to

$$
(1-M \sigma)^{2}+\beta^{3} Z \gamma=0 .
$$

As $\gamma$ was defined with negative imaginary part, the asymptotics of $\operatorname{Im}(\Omega \gamma)$ large positive does not occur. 


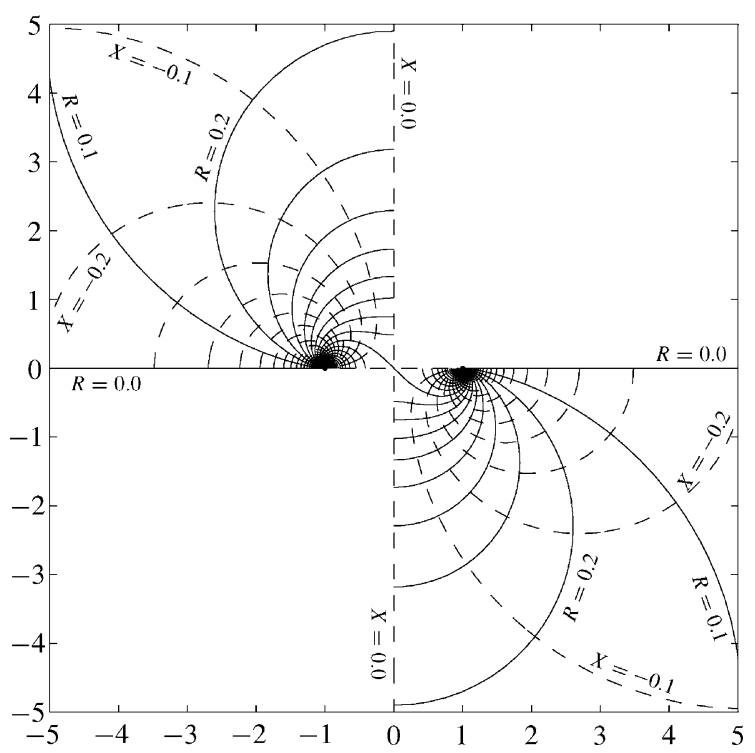

Fig. 2. Trajectories of surface waves $\sigma$ (Eq. (10b)) for varying $Z=R+\mathrm{i} X ; M=0$. Fixed $R$ and $X=0:-0.1:-\infty$ (一). Fixed $X$ and $R=0: 0.1: \infty(---)$.

If $\Omega$ is large, which is the typical situation in aircraft engine duct problems, any solution of (11)—except for impedances near the bordering lines-produces a large $\operatorname{Im}(\Omega \gamma)$ and is therefore an approximation of a solution of (8b).

The physical interpretation of these solutions is again that the corresponding mode is spatially confined to the immediate neighbourhood of the wall, as the modal shape function $J_{m}(\Omega \gamma r)$ becomes exponentially decaying away from the wall (Eq. (A.1)):

$$
\left|\frac{J_{m}(\Omega \gamma r)}{J_{m}(\Omega \gamma)}\right| \simeq \frac{\mathrm{e}^{\Omega \operatorname{Im}(\gamma)(1-r)}}{r^{1 / 2}} .
$$

In other words, these modes are not really duct modes, but surface waves. ${ }^{4}$ By bringing the second term to the right and squaring both sides, Eq. (11) becomes a polynomial in $\sigma$ of degree 4. It follows that there are at most four such surface waves. Their existence and number varies with $Z$ and $M$.

The other possible solutions of Eqs. (8a) and (8b), the ones with $\operatorname{Im}(\Omega \gamma)$ not large, necessarily occur near (i.e. typically within a distance $\mathcal{O}\left(\omega^{-1}\right)$ ) the branch cuts of square root $\gamma(\sigma)$, where $\operatorname{Im}(\gamma)=0$.

Trajectories of these surface wave numbers given by Eq. (11), as function of $Z$, are plotted in Fig. 4. To include all complex values of $Z$, we have drawn two families of curves: one for fixed $\operatorname{Re}(Z)$ and one for fixed $\operatorname{Im}(Z)$. Un-attenuated waves occur for purely imaginary $Z$, but in contrast to the no-flow case, such $Z$ do allow also attenuated modes.

An interesting special case is the incompressible flow limit $(M \rightarrow 0, M \sigma$ fixed, $Z=\mathcal{O}(M))$, where the solutions can be given explicitly. Define $S=M \sigma, \zeta=Z / 2 M$, then we get

$$
(1-S)^{2}-2 \mathrm{i} \zeta \operatorname{Re}(S) S=0,
$$

\footnotetext{
${ }^{4}$ We note in passing that boundary condition (3) is based on the assumption of a thin mean flow boundary layer, which should of course remain thin compared to the surface wave penetration depth $\operatorname{Im}(\Omega \gamma)^{-1}$.
} 
126

S.W. Rienstra/Wave Motion 37 (2003) 119-135
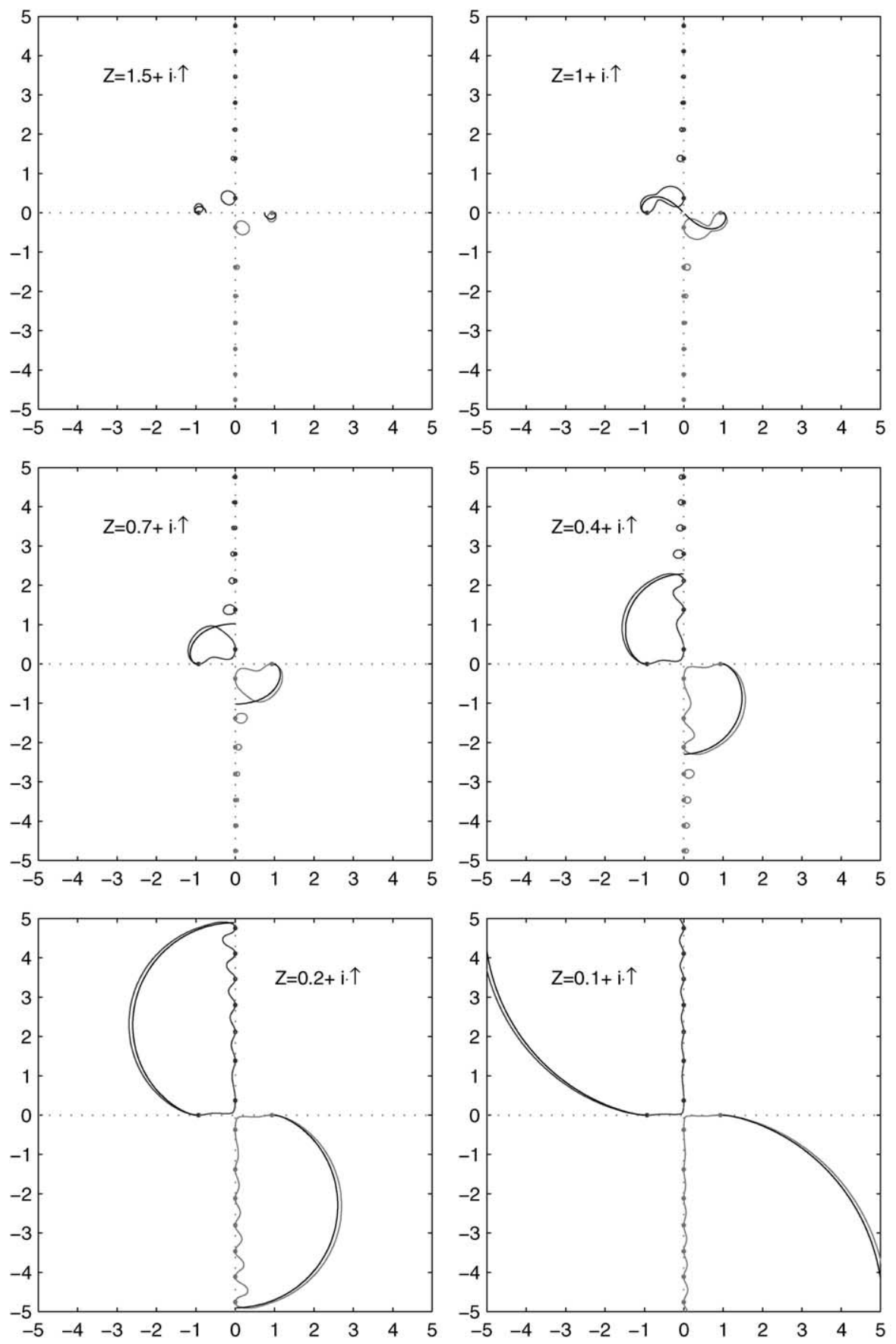

Fig. 3. Trajectories of $\sigma$ for $m=1, \omega=5, M=0,-\infty<\operatorname{Im}(Z)<\infty$. 


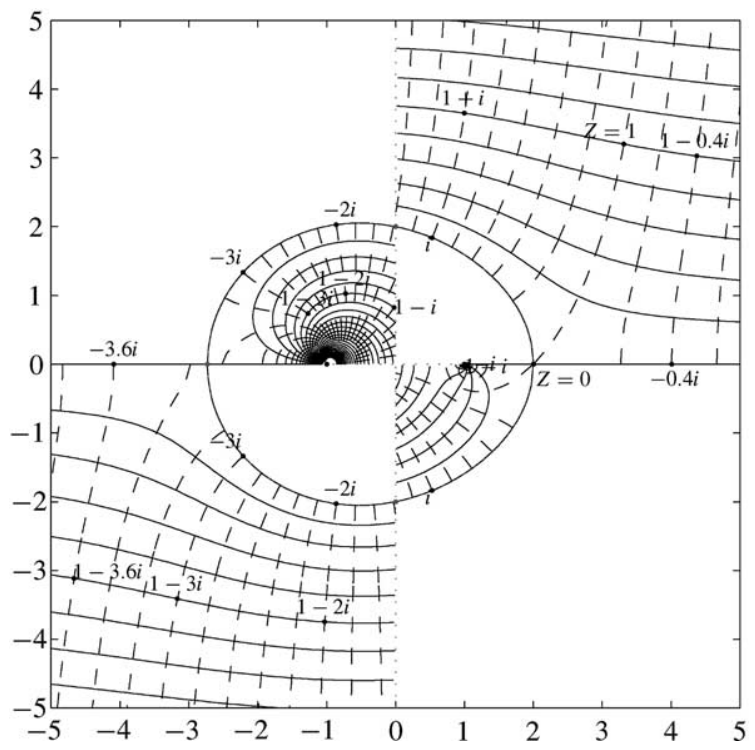

Fig. 4. Trajectories of surface waves $\sigma$ (Eq. (11)) for varying $Z=R+\mathrm{i} X ; M=0.5$. (Some specific values of $Z$ are indicated.) Fixed $R$ and $X=-\infty: 0.2: \infty(-)$. Fixed $X$ and $R=0: 0.2: \infty(--)$.

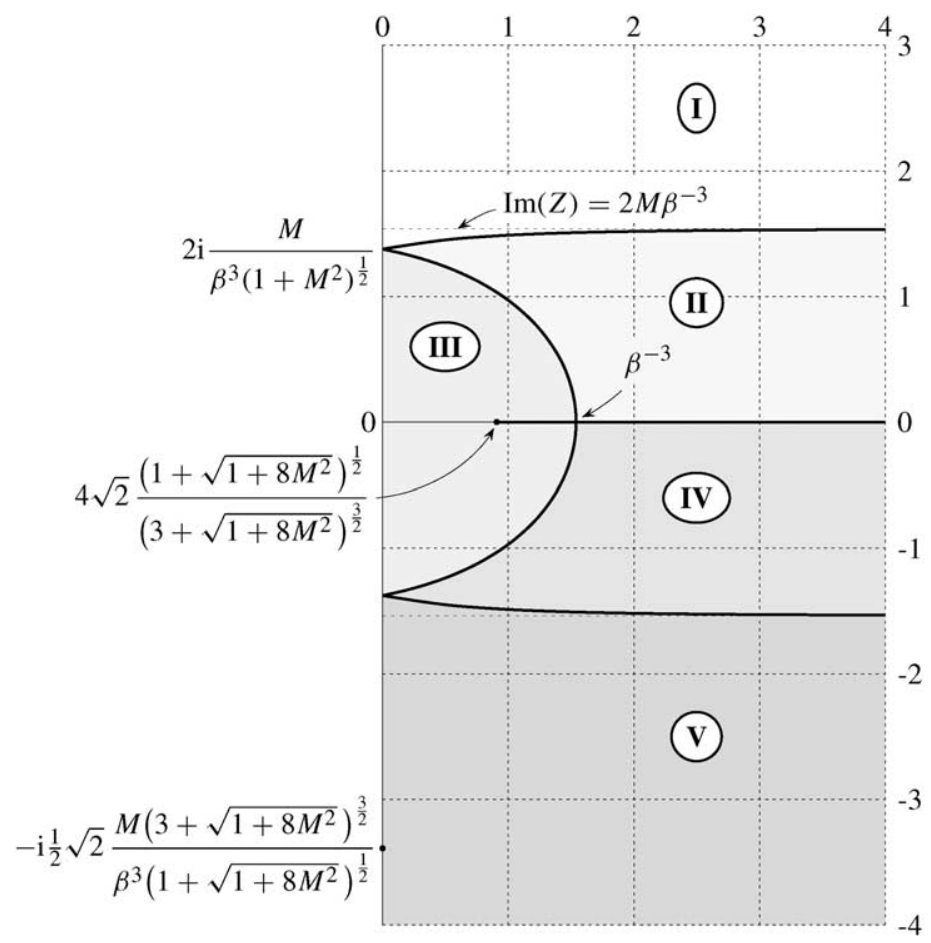

Fig. 5. Complex $Z$-plane, with regions of different numbers of surface waves. No solutions in $\mathbf{I}, \sigma_{\mathrm{HI}} \in \mathbf{I I}, \ldots, \mathbf{V}, \sigma_{\mathrm{SR}} \in \mathbf{I I I}, \ldots, \mathbf{V}$, $\sigma_{\mathrm{SL}} \in \mathbf{I V}, \ldots, \mathbf{V}, \sigma_{\mathrm{HS}} \in \mathbf{V}$. Thick lines map to the branch cuts in Fig. 6 . In the figure, $M=0.5$ is taken. 
with four solutions (one in each quadrant)

$$
\begin{aligned}
& S_{\mathrm{HI}}=\mathrm{ie}^{\operatorname{arsinh}(\zeta-\mathrm{i})}, \quad \operatorname{Im}(\zeta) \leq 1, \\
& S_{\mathrm{SR}}=-\mathrm{ie}^{-\operatorname{arsinh}(\zeta-\mathrm{i})}, \quad \operatorname{Im}(\zeta) \leq 1, \\
& S_{\mathrm{HS}}=-\mathrm{ie}^{\operatorname{arsinh}(\zeta+\mathrm{i})}, \quad \operatorname{Im}(\zeta) \leq-1, \\
& S_{\mathrm{SL}}=\mathrm{ie}^{-\operatorname{arsinh}(\zeta+\mathrm{i})}, \quad \operatorname{Im}(\zeta) \leq-1 .
\end{aligned}
$$

We may now apply, as in [15], the causality arguments of [16,17], and vary the frequency $\omega$ to become negative imaginary, i.e. $\omega=-\mathrm{i} v$. A typical impedance of mass-spring-damper type then yields $\zeta \sim r+\mathrm{i} a \omega-\mathrm{i} b / \omega=r+a v+$ $b / v$, which is real. As a result, the above wave number $\omega M^{-1} S_{\mathrm{HI}}$ shifts to the lower half of the complex plane, which means that it denotes a right-running, growing wave and therefore an instability. So for at least some impedances and parameter values, this surface wave of the first quadrant is an instability. Therefore, we have tentatively called it "hydrodynamic instability" $\sigma_{\mathrm{HI}}$. The others are called: a right-running stable hydrodynamic surface wave $\sigma_{\mathrm{HS}}$, a right-running ordinary surface wave $\sigma_{\mathrm{SR}}$ and a left-running ordinary surface wave $\sigma_{\mathrm{SL}}$. ("Hydrodynamic" because they exist only with flow.)

Solutions of Eq. (11) may be analysed in great detail, and the results are summarised in the Figs. 5 and 6, and Table 1 . The only impedances that may possibly occur (for passive walls) are with $\operatorname{Re}(Z) \geq 0$. So the imaginary $Z$-axis is an important borderline, which is mapped (as far as it allows solutions) to the $\sigma$-plane as the

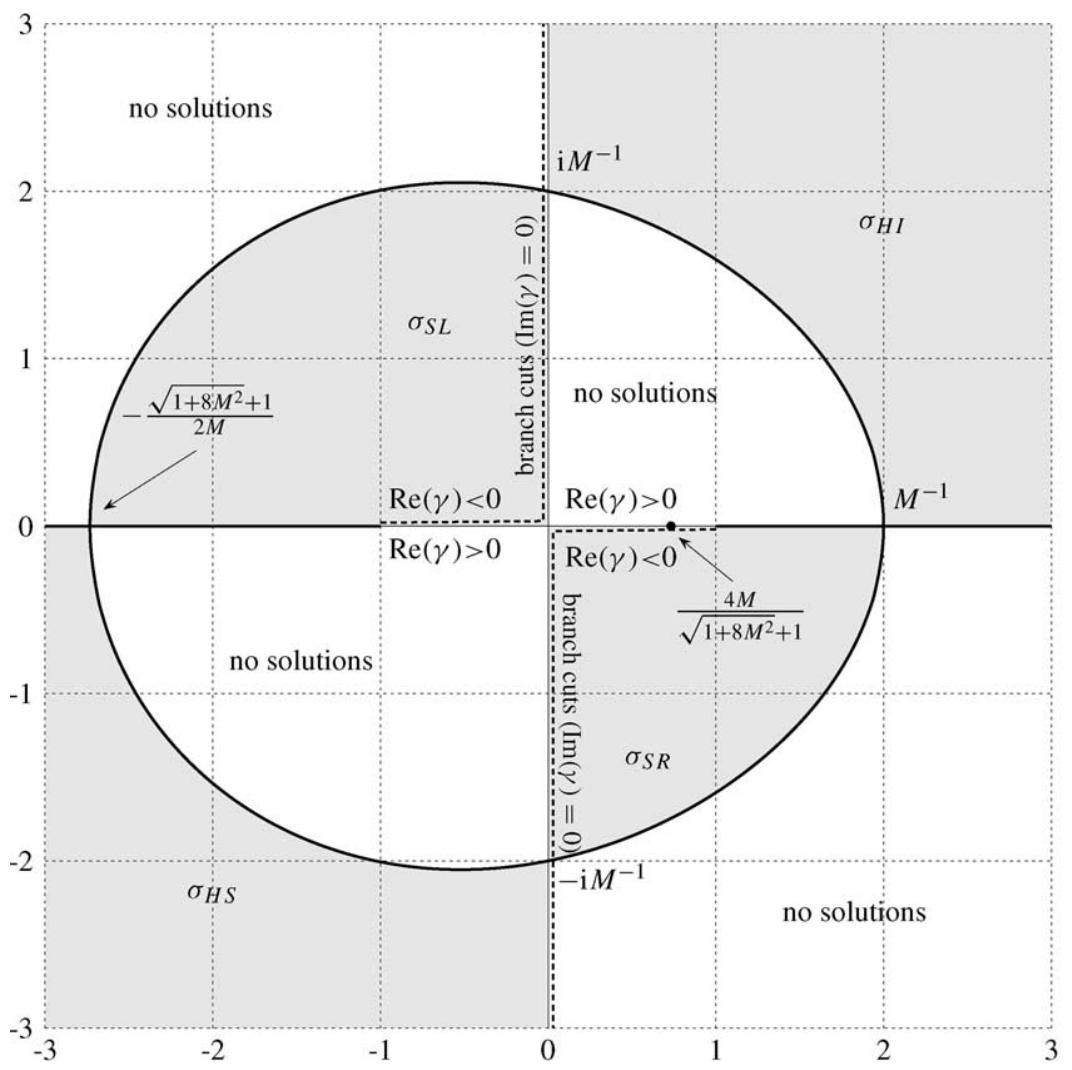

Fig. 6. Regions of existence of surface wave $\sigma$. Thick lines map to the imaginary $Z$-axis in Fig. 5 (except the part in region $\mathbf{I}$ where no solutions exist). In the figure, $M=0.5$ is taken. 
Table 1

Particular values of $Z$ and $\sigma$, satisfying Eq. (11)

\begin{tabular}{|c|c|c|c|c|}
\hline$Z$ & $\sigma_{\mathrm{HI}}$ & $\sigma_{\mathrm{SR}}$ & $\sigma_{\mathrm{SL}}$ & $\sigma_{\mathrm{HS}}$ \\
\hline$\infty+2 \mathrm{i} \frac{M}{\beta^{3}}-\mathrm{i} 0$ & $0+\mathrm{i} \infty$ & $-^{\mathrm{b}}$ & - & - \\
\hline $2 \mathrm{i} \frac{M}{\beta^{3} \sqrt{1+M^{2}}}-\mathrm{i} 0$ & $\frac{\mathrm{i}}{M}$ & $-\frac{\mathrm{i}}{M}$ & - & - \\
\hline 0 & $\frac{1}{M}$ & $\frac{1}{M}$ & - & - \\
\hline $4 \sqrt{2} \frac{\left(1+\sqrt{1+8 M^{2}}\right)^{1 / 2}}{\left(3+\sqrt{1+8 M^{2}}\right)^{3 / 2}}$ & $\begin{array}{l}\frac{5-\sqrt{1+8 M^{2}}}{2 M} \\
+\mathrm{i} \frac{\left(1-10 M^{2}+\left(2 M^{2}+1\right) \sqrt{1+8 M^{2}}\right)^{1 / 2}}{\sqrt{2} M^{2}}\end{array}$ & $\frac{4 M}{1+\sqrt{1+8 M^{2}}}$ & - & - \\
\hline$\frac{1}{\beta^{3}}+0+\mathrm{i} 0$ & $\ldots{ }^{c}$ & - & - & - \\
\hline$\frac{1}{\beta^{3}}-0+\mathrm{i} 0$ & $\ldots$ & 0 & - & - \\
\hline$\frac{1}{\beta^{3}}-0-\mathrm{i} 0$ & $\cdots$ & $\cdots$ & - & - \\
\hline$\frac{1}{\beta^{3}}+0-\mathrm{i} 0$ & $\ldots$ & $\ldots$ & 0 & - \\
\hline$\infty-$ i0 & $\frac{2}{M}+\mathrm{i} \infty$ & 1 & -1 & - \\
\hline$-2 \mathrm{i} \frac{M}{\beta^{3} \sqrt{1+M^{2}}}-\mathrm{i} 0$ & $\frac{2}{M}+\frac{1}{M}\left(\frac{3-M^{2}}{1+M^{2}}\right)^{1 / 2}$ & $\frac{2}{M}-\frac{1}{M}\left(\frac{3-M^{2}}{1+M^{2}}\right)^{1 / 2}$ & $\frac{\mathrm{i}}{M}$ & $-\frac{\mathrm{i}}{M}$ \\
\hline$\infty-2 \mathrm{i} \frac{M}{\beta^{3}}-\mathrm{i} 0$ & $\frac{4}{M}+\mathrm{i} \infty$ & 1 & -1 & $-\mathrm{i} \infty$ \\
\hline$-\mathrm{i} \frac{1}{2} \sqrt{2} \frac{M\left(3+\sqrt{1+8 M^{2}}\right)^{3 / 2}}{\beta^{3}\left(1+\sqrt{1+8 M^{2}}\right)^{1 / 2}}$ & \multicolumn{2}{|c|}{$\underline{5+\sqrt{1+8 M^{2}}}+\underline{\left(10 M^{2}-1+\left(2 M^{2}+1\right) \sqrt{1+8 M^{2}}\right)^{1 / 2}}$} & $-\frac{1+\sqrt{1+8 M^{2}}}{2 M}$ & $-\frac{1+\sqrt{1+8 M^{2}}}{2 M}$ \\
\hline
\end{tabular}

${ }^{\text {a }}$ Note that a $Z$ crossing the real segment $\left[4 \sqrt{2}\left(1+\sqrt{1+8 M^{2}}\right)^{1 / 2} /\left(3+\sqrt{1+8 M^{2}}\right)^{3 / 2}, 1 / \beta^{3}\right]$ in $Z$-plane, causes $\sigma_{\mathrm{SR}}$ to disappear and-at the same time-reappear on either side of $\sigma=4 M /\left(1+\sqrt{1+8 M^{2}}\right)$ along the real segment $[0,1]$ in $\sigma$-plane.

${ }^{b}$ No solution exist.

${ }^{\mathrm{c}}$ Expressions too complicated to be of interest. 

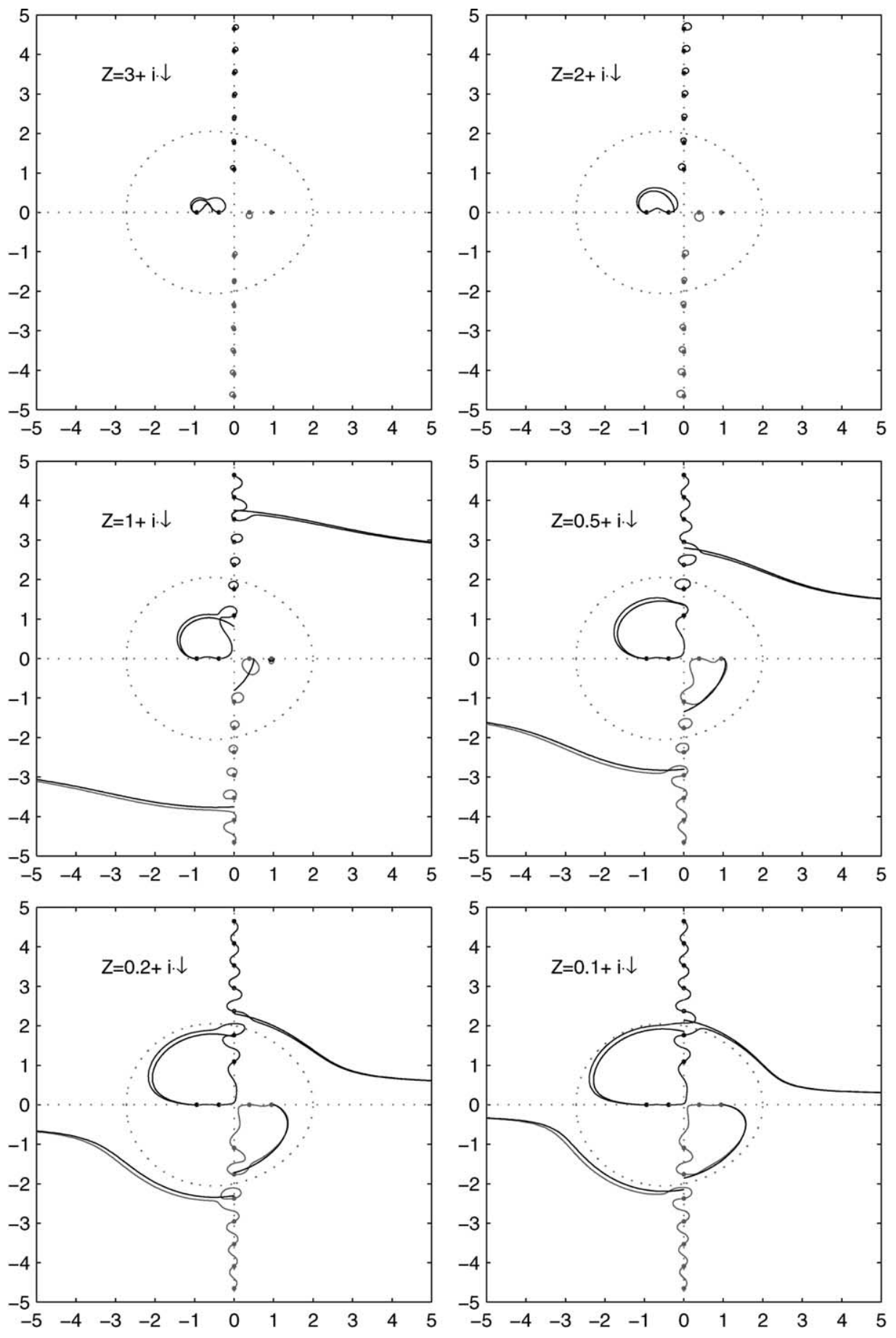

Fig. 7. Trajectories of $\sigma$ for $m=1, \omega=5, M=0.5,-\infty<\operatorname{Im}(Z)<\infty$. 
egg-shaped contour and the part of real axes given by $(-\infty,-1) \cup(1, \infty)$. In the $\sigma$-plane the branch cuts of $\gamma$ are important borderlines, where the $2 \mathrm{D}$ approximation breaks down, and the solutions become regular duct modes again. (In the approximation they disappear to the other Riemann sheet.) The branch cuts are mapped to the $Z$-plane as the four contours that separate the five regions of existence of different numbers of surface waves.

The egg in the axial wave number plane plays a prominent role, which is not entirely unexpected, as the typical radius $M^{-1}$ in reduced wave number $\sigma$ corresponds approximately with the Strouhal number $\omega / M$ in dimensional wave numbers $k$ and with the hydrodynamic wave number $\tilde{\omega} / U_{0}$ to the dimensionless wave numbers.

Trajectories of all duct mode eigenvalues are plotted in Fig. 7, as a function of the imaginary part $X$ of impedance $Z=R+\mathrm{i} X$, and various $R$, for $\omega=5, m=1$, and $M=0.5$. For illustration, the 2D surface wave approximations are included, and the agreement is seen to be very good. As in Fig. 3, if we used a much higher $\omega$, the agreement would have been too good, with hardly any visible difference. This figure is further discussed in the following section. The reason why we let $\operatorname{Im}(Z)=X$ decrease is explained in Section 6 .

\section{Further observations}

\subsection{Qualitative behaviour as function of $Z$ and $M$}

Suppose we vary the impedance from hard-wall to hard-wall, via vertical straight lines in the complex plane: $Z=R+\mathrm{i} X$, where $R$ is fixed and $X$ varies between $-\infty$ and $\infty$.

Without mean flow (Fig. 3), most eigenvalues return to the hard-wall value they started from. Some, however, form a closed loop such that they meander from their initial hard-wall value to the next hard-wall value. The loop is closed by the first eigenvalue, which becomes a surface wave (when $X$ increases from $-\infty$ ) and, following a large circular contour, turns back (when $X \simeq 0$ ) to a hard-wall eigenvalue of much higher index. Since $\omega \gamma=-\omega / R \simeq-j_{m \mu}^{\prime}$, the hard-wall return-index $\mu_{\text {ret }}$ can be estimated as (see (A.3))

$$
\mu_{\text {ret }}=\frac{\omega}{\pi R}-\frac{1}{2} m+\frac{1}{4}
$$

This circular loop becomes larger and larger when $R$ tends to zero, until it becomes unbounded for $R=0$. It shrinks to zero when $R$ becomes large.

With mean flow (Fig. 7), the situation is quite different. Not some, but most of the eigenvalues move up one position. When $R$ is big enough, the acoustic surface waves (within the egg; typically $|\sigma|<M^{-1}$ ) form a closed loop. This loop does not grow to infinity when $R$ tends to zero, but grows until it approximately coincides with the egg. The hydrodynamic surface waves (outside the egg ${ }^{5}$ typically $|\sigma|>M^{-1}$ ) on the other hand, start at a hard-wall value at $X=\infty$, but tends to infinity as follows

$$
\sigma \simeq \pm \frac{\mathrm{i} \beta^{3} Z+2 M}{M^{2}}= \pm \frac{-\beta^{3} X+\mathrm{i} \beta^{3} R+2 M}{M^{2}} \quad(X \rightarrow-\infty),
$$

so they disappear to infinity along lines parallel to the real axis. Only the finite number of modes between the acoustic and hydrodynamic surface waves return to their initial hard-wall values.

Following whatever contour in $Z$, the modes inside the egg remain practically inside, and the modes outside remain outside $^{6}$. So there are only a finite number of hard-wall modes that may turn into an acoustic surface wave, while the others may become a hydrodynamic surface wave. The hard-wall modal index $\mu_{\text {crit }}$ separating these modes may be estimated from the fact that the egg crosses the imaginary axis at $\sigma=\mp \mathrm{i} M^{-1}$, or $\Omega \gamma \simeq-\Omega \sqrt{1+M^{-2}}=-j_{m \mu}^{\prime}$.

\footnotetext{
${ }^{5}$ Note that for $M \rightarrow 0$, the egg becomes infinitely large, pushing the hydrodynamic surface waves away to infinity.

${ }^{6}$ Apart from rare exceptions when $R$ is small.
} 
This yields approximately (see (A.3))

$$
\mu_{\mathrm{crit}} \simeq \frac{\omega}{\pi M \beta} \sqrt{1+M^{2}}-\frac{1}{2} m+\frac{1}{4} .
$$

\subsection{Multi-valued functions and branch points}

The above analysis is mainly descriptive, and the results are interpreted with emphasis on their physical context. The deeper origin of this strange game of musical chairs may therefore remain in the background.

From a complex-function point of view, it is not so strange. If we consider Eqs. (8a) and (8b) or (9a) and (9b) as the definition of a complex function $\sigma=\sigma(Z)$ of complex variable $Z$, this function is evidently multi-valued with an infinite number of branches: each branch of $\sigma(Z)$ represents a mode. The branch points-the points where two or more branches coalesce-are found at the $Z$-values where the $\sigma$-derivative of Eq. (8b) or (9b) vanishes (also known as the impedances with double eigenvalues). If we follow a closed contour in the complex $Z$-plane (the contour may be closed at infinity) such that a branch point is encircled, we arrive at another branch (i.e. another eigenvalue) when we return to the $Z$ we started from.

This is exactly what happens when we trace the contours $Z=R+\mathrm{i} X$ ( $R$ fixed) for smaller and smaller $R$. We start and end at the same $Z=\infty$, but the smaller the $R$, the more double eigenvalues (branch points) are encircled, and the eigenvalue we return to moves higher up in the list.

\section{How to find all eigenvalues by continuation in $Z$ ?}

Based on the above-described dynamics of the eigenvalues in the complex $Z$-plane, we can devise a continuation method to find all eigenvalues for given $M, m, \omega$ and $Z$.

Assume that the hard-wall values are known. Of course, some effort is to be invested here too, but since all (reduced) radial eigenvalues $\Omega \gamma_{m \mu}=j_{m \mu}^{\prime}$ are real, independent of $M$, and asymptotically for large $\mu$ found at fixed intervals, this is relatively easy. Now we can connect a contour in the complex $Z$-plane from any $Z=\infty$ to the sought value. Then we can trace the eigenvalues as a function of $Z$ as follows. Start at a large enough $Z$-value, take small enough steps along the contour, use the previous values as starting values, and solve by a simple Newton-Raphson zero-finding routine the eigenvalue equation at each $Z$-position.

As we have seen, in some parts of the $Z$-plane we have a problem: surface waves may disappear to or appear from infinity. When they come in from infinity, we have to make sure to pick up these eigenvalues somewhere, which may be not so easy. It is therefore easier to take a starting " $Z=\infty$ " without disappearing surface waves. We propose contours parallel to the imaginary axis, like was done in Figs. 3 and 7. For $M=0$, this is $Z=$ $R-\mathrm{i} \infty$, while for $M>0$ this is $Z=R+\mathrm{i} \infty$ (note the corresponding up- and down-arrows given in the figures.)

For $R=0$, some care is required when upper and lower half plane solutions meet along the real $\sigma$-axis, for example at $\sigma=0$ and $M^{-1}$.

\section{Exact results}

Just for the record, a few exact results on the existence of surface waves can be given. Note that for imaginary $Z=\mathrm{i} X$ and imaginary radial wave numbers $\gamma=-\mathrm{i} \tau$ (and $\sigma$ real) Eq. (8b) may be rewritten as the real equation

$$
\frac{\Omega \tau I_{m+1}(\Omega \tau)}{I_{m}(\Omega \tau)}=-m-\frac{\Omega}{\beta^{3} X}(1-M \sigma)^{2} .
$$




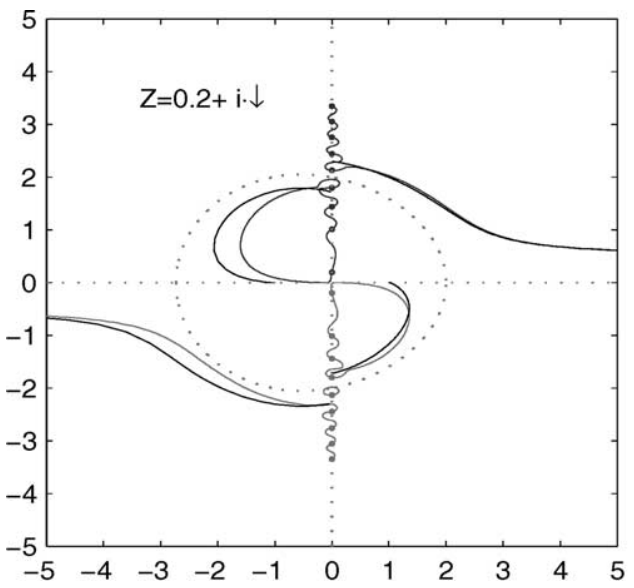

Fig. 8. Trajectories of $\sigma$ for $m=\omega=10, M=0.5$, and varying $Z=0.2+\mathrm{i} X$.

Since $z I_{m+1}(z) / I_{m}(z)$ is monotonically increasing in $z \geq 0$, we have for $M=0$ exactly two solutions if $-\omega / m \leq$ $X<0$ and none otherwise. If $M \neq 0$, we have no solutions if $X>0$, and maybe up to 4 otherwise. There is at least one if $X<-\Omega(1 \pm M)^{2} / \beta^{3} m$.

\section{Large circumferential order $m$}

The used asymptotic expression (A.1) of $J_{m}$ applies only for fixed $m$, and is totally inappropriate for $m \geq \mathcal{O}(\omega)$. This, however, does not necessarily mean that the surface wave behaviour immediately disappears, because only the limit $\left|J_{m}^{\prime} / J_{m}\right| \rightarrow \mathcal{O}(1)$ was essential. It appears that for $m \sim \omega$ the general behaviour remains the same, in particular the occurrence of surface waves. See Fig. 8. For $m>\mathcal{O}(\omega)$, a certain surface wave behaviour may still be recognised, but not any more described by the above equations.

\section{The annular duct}

For the annular duct with dimensionless hub radius $h$ and an impedance $Z_{1}$ at $r=1$ and $Z_{h}$ at $r=h$ we find the reduced eigenvalue equation

$$
\begin{aligned}
& (1-M \sigma)^{4}\left(J_{m}(\Omega \gamma) Y_{m}(\Omega \gamma h)-Y_{m}(\Omega \gamma) J_{m}(\Omega \gamma h)\right) \\
& \quad+\mathrm{i} \beta^{3} Z_{h} \gamma(1-M \sigma)^{2}\left(J_{m}(\Omega \gamma) Y_{m}^{\prime}(\Omega \gamma h)-Y_{m}(\Omega \gamma) J_{m}^{\prime}(\Omega \gamma h)\right) \\
& \quad-\mathrm{i} \beta^{3} Z_{1} \gamma(1-M \sigma)^{2}\left(J_{m}^{\prime}(\Omega \gamma) Y_{m}(\Omega \gamma h)-Y_{m}^{\prime}(\Omega \gamma) J_{m}(\Omega \gamma h)\right) \\
& \quad+\beta^{6} Z_{1} Z_{h} \gamma^{2}\left(J_{m}^{\prime}(\Omega \gamma) Y_{m}^{\prime}(\Omega \gamma h)-Y_{m}^{\prime}(\Omega \gamma) J_{m}^{\prime}(\Omega \gamma h)\right)=0,
\end{aligned}
$$

where $Y_{m}$ is the $m$ th order Bessel function of the 2nd kind. By substituting the asymptotic approximation (A.2) (and similar for the forms with derivatives), we obtain the surface wave equation

$$
\left((1-M \sigma)^{2}+\beta^{3} Z_{1} \gamma\right)\left((1-M \sigma)^{2}+\beta^{3} Z_{h} \gamma\right)=0
$$

which is just the product of the surface wave equations at inner and outer duct wall. Depending on the selected $Z_{1}$ and $Z_{h}$, the mode may be of surface wave type at either or both duct walls. Therefore, occurrence and behaviour of any surface waves is similar to what is presented above. 


\section{Conclusions}

An analysis is made of the behaviour of the acoustic modes of a lined duct with and without uniform mean flow. The lining is assumed to be locally reacting and of impedance type, while the frequency of the sound field is fixed and given. It is shown that for high dimensionless frequencies $\omega$ (as prevail in lined flow ducts of turbofan engines) and given circumferential order $m \leq \mathcal{O}(\omega)$ we have three types of modes.

(i) An infinite number of genuine acoustic modes, of which the complex axial wave number, as a function of wall impedance $Z$, is never far away from the hard-wall values.

(ii) Two acoustic surface waves, which occur both with and without mean flow.

(iii) Two hydrodynamic surface waves, which occur only with mean flow.

Surface waves are called that way because their field is only significant close to the wall, as it decays exponentially away from the wall. They are essentially 2D and independent of the duct geometry. The governing equation is therefore much simpler than for the general duct mode, and allows a detailed analysis.

The surface waves exist only when $Z$ is in certain areas of the complex $Z$-plane, which are given in detail. The reduced axial wave number of the surface waves can be found in the complex plane in very specific areas, separated by an egg-shaped border with a typical radius equal to the hydrodynamic wave number. The acoustic surface waves are found inside the egg in the 2 nd and 4th quadrant $\left(M>0, \mathrm{e}^{+\mathrm{i} \omega t}\right.$-convention), and the hydrodynamic surface waves are found outside the egg in the 1st and 3rd quadrant. At least in the incompressible limit and for certain type of impedances, one hydrodynamic surface waves can be shown to be an instability. It is expected that this remains true in more general cases.

Relevant for eigenvalue searching routines is the following observation. When a mode is traced along a path in the complex $Z$-plane, for example from hard-wall value to hard-wall value a contour parallel to the imaginary axis, the mode does not always return to its original value but changes position with another mode. This happens when one or more branch points of the axial wave number, considered as a function of $Z$, are encircled. These branch points correspond to the impedances with double eigenvalues.

\section{Appendix A. Bessel functions}

We have for fixed order $m$ and large values of argument $z$ in the lower half of the complex plane [13, formula 9.2]

$$
J_{m}(z) \simeq \frac{\mathrm{e}^{\mathrm{i} z-\mathrm{i} m \pi / 2-\mathrm{i} \pi / 4}}{\sqrt{2 \pi z}}, \quad z \rightarrow \infty, \operatorname{Im}(z)<0
$$

and similarly

$$
J_{m}(z) Y_{m}(z h)-Y_{m}(z) J_{m}(z h) \simeq \frac{\mathrm{i}}{\pi z \sqrt{h}} \mathrm{e}^{\mathrm{i}(1-h) z}, \quad z \rightarrow \infty, \operatorname{Im}(z)<0 .
$$

Note that for our surface wave classification we essentially deal here with complex values of the argument, while the exponential growth of $J_{m}$ is the key factor. For real $z$ and $m \neq 0$ far better expressions are available due to Debije [13, (formulae 9.3), 18]. However, these expressions do not explicitly yield the zeros $j_{m \mu}^{\prime}$. Therefore, we still use for our estimates of which modes reside inside or outside the egg the relatively crude approximations

$$
j_{m \mu}^{\prime} \simeq\left(\mu+\frac{1}{2} m-\frac{1}{4}\right) \pi, \quad \mu \rightarrow \infty .
$$

\section{References}

[1] J.M. Tyler, T.G. Sofrin, Axial flow compressor noise studies, Trans. Soc. Automot. Eng. 70 (1962) 309-332.

[2] W.E. Zorumski, Acoustic theory of axisymmetric multi-sectioned ducts, NASA TR R-419, 1974. 
[3] B.J. Tester, The optimization of modal sound attenuation in ducts, in the absence of mean flow, J. Sound Vib. 27 (4) (1973) $477-513$.

[4] B.J. Tester, The propagation and attenuation of sound in ducts containing uniform or "plug" flow, J. Sound Vib. 28 (2) (1973) $151-203$.

[5] B.J. Tester, Some aspects of sound attenuation in lined ducts containing inviscid mean flows with boundary layers, J. Sound Vib. 28 (2) (1973) 217-245.

[6] W. Koch, W. Möhring, Eigensolutions for liners in uniform mean flow ducts, AIAA J. 21 (1983) 200-213.

[7] S.W. Rienstra, The acoustics of a lined duct with flow, NLR TR 87002 U, 1987.

[8] E.R. Rademakers, Experimental validation of the lined duct acoustics model LINDA, NLR CR 89236L, 1989.

[9] S.W. Rienstra, Sound transmission in slowly varying circular and annular ducts with flow, J. Fluid Mech. 380 (1999) $279-296$.

[10] S.W. Rienstra, W. Eversman, A numerical comparison between multiple-scales and FEM solution for sound propagation in lined flow ducts, J. Fluid Mech. 437 (2001) 367-384.

[11] K.U. Ingard, Influence of fluid motion past a plane boundary on sound reflection, absorption, and transmission, J. Acoust. Soc. Am. 31 (7) (1959) 1035-1036.

[12] M.K. Myers, On the acoustic boundary condition in the presence of flow, J. Sound Vib. 71 (3) (1980) 429-434.

[13] M. Abramowitz, I.A. Stegun, Handbook of Mathematical Functions, National Bureau of Standards, Dover, New York, 1964.

[14] M.C. Quinn, M.S. Howe, On the production and absorption of sound by lossless liners in the presence of mean flow, J. Sound Vib. 97 (1) (1984) 1-9.

[15] S.W. Rienstra, Hydrodynamic instabilities and surface waves in a flow over an impedance wall, in: G. Comte-Bellot, J.E. Ffowcs Williams (Eds.), Proceedings of the IUTAM Symposium 'Aero- and Hydro-Acoustics', 1985, Lyon, Springer, Heidelberg, 1986, pp. $483-490$.

[16] D.S. Jones, J.D. Morgan, The instability of a vortex sheet on a subsonic stream under acoustic radiation, Proc. Camb. Phil. Soc. 72 (1972) 465-488.

[17] D.G. Crighton, F.G. Leppington, Radiation properties of the semi-infinite vortex sheet: the initial-value problem, J. Fluid Mech. 64 (2) (1974) 393-414.

[18] F.W.J. Olver, Asymptotics and Special Functions, Academic Press, New York, 1974. 